\title{
Monitoring abacavir bioactivation in humans: Screening for an aldehyde metabolite
}

\author{
Nádia M. Grilo a Alexandra M.M. Antunes $^{\mathrm{b}, *}$, Umbelina Caixas ${ }^{\mathrm{a}, \mathrm{c}}$, Aline T. Marinho ${ }^{\mathrm{a}}$, \\ Catarina Charneira $^{\mathrm{b}}$, M. Conceição Oliveira $^{\mathrm{b}}$, Emília C. Monteiro ${ }^{\mathrm{a}}$, M. Matilde Marques ${ }^{\mathrm{b}}$, \\ Sofia A. Pereira ${ }^{\mathrm{a}, * *}$ \\ a Centro de Estudos de Doenças Crónicas (CEDOC), Faculdade de Ciências Médicas (FCM), Universidade Nova de Lisboa, 1169-056 Lisboa, Portugal \\ b Centro de Química Estrutural (CQE), Instituto Superior Técnico, Universidade Técnica de Lisboa, 1049-001 Lisboa, Portugal \\ ${ }^{\mathrm{c}}$ Centro Hospitalar de Lisboa Central (CHLC), 1150-199 Lisboa, Portugal
}

\section{H I G H L I G H T S}

- We identified abacavir-protein adducts in the hemoglobin of HIV-infected patients.

- Abacavir-protein adducts might be used as biomarkers of $\mathrm{ABC}$-induced toxicity.

- We present the first evidence of abacavir-protein adduct formation in humans.

- The data provide clues to the mechanisms underlying abacavir adverse reactions.

\section{A R T I C L E I N F O}

\section{Article history:}

Received 22 January 2013

Received in revised form 21 February 2013

Accepted 23 February 2013

Available online 4 March 2013

\section{Keywords:}

Abacavir aldehyde

Hypersensitivity syndrome

Cardiotoxicity

Biomarkers of drug bioactivation

\section{G R A P H I C A L A B S T R A C T}

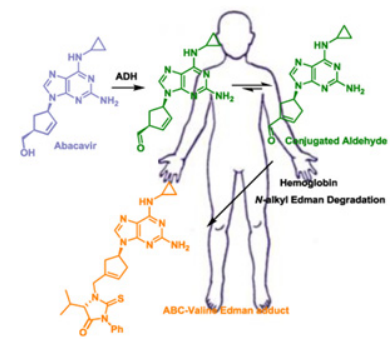

\begin{abstract}
A B S T R A C T
The anti-HIV drug abacavir is associated with idiosyncratic hypersensitivity reactions and cardiotoxicity. Although the mechanism underlying abacavir-toxicity is not fully understood, drug bioactivation to reactive metabolites may be involved. This work was aimed at identifying abacavir-protein adducts in the hemoglobin of HIV patients as biomarkers of abacavir bioactivation and protein modification. The protocol received prior approval from the Hospital Ethics Committee, patients gave their written informed consent and adherence was controlled through a questionnaire. Abacavir-derived Edman adducts with the $\mathrm{N}$-terminal valine of hemoglobin were analyzed by an established liquid chromatography-electrospray ionization-tandem mass spectrometry method. Abacavir-valine adducts were detected in three out of ten patients. This work represents the first evidence of abacavir-protein adduct formation in humans. The data confirm the ability of abacavir to modify self-proteins and suggest that the molecular mechanism(s) of some abacavir-induced adverse reactions may require bioactivation.
\end{abstract}

(C) 2013 Elsevier Ireland Ltd. All rights reserved.

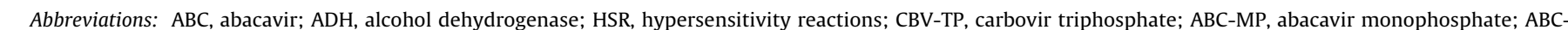

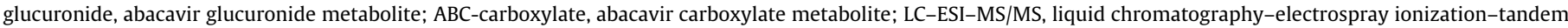
mass spectrometry; MI, myocardial infarction.

* Corresponding author at: Centro de Química Estrutural(CQE), Complexo I, Instituto Superior Técnico, Av. Rovisco Pais, 1, $1049-001$ Lisboa, Portugal. Tel.: +351 21 8417627; fax: +351218464455.

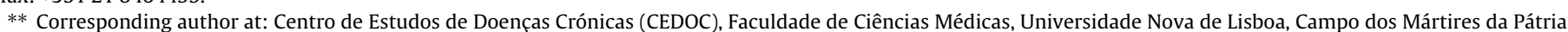
130, 1169-056 Lisboa, Portugal. Tel.: +351 21 8803000; fax: +351 218851920.

E-mail addresses: alexandra.antunes@ist.utl.pt (A.M.M. Antunes), sofia.pereira@fcm.unl.pt (S.A. Pereira). 


\section{Introduction}

The anti-HIV drug abacavir (ABC; Scheme 1$)$ is associated with hypersensitivity reactions (HSRs), characterized by systemic symptoms that include fever, rash, nausea, vomiting, abdominal pain, lethargy and malaise. This allergenic response occurs generally within the first 6 weeks of treatment and has been linked to HLA class I allele HLA-B*57:01 (Nolan, 2009). In addition, long-term abacavir exposure has been associated with an increased risk of myocardial infarction (Costagliola et al., 2010; Islam et al., 2012) that prompted the FDA to issue an alert for heart attack risk in March 2011 (FDA, 2011). The potential for cardiotoxicity raises further concerns about chronic use of the drug and calls for urgent and accurate risk/benefit estimations. Toward this goal, both a thorough understanding of the mechanisms of abacavir-induced toxicity and the development of reliable toxicity biomarkers are required.

Four different models/hypotheses have been considered to explain the mechanism of the immune-mediated adverse reactions induced by abacavir: (1) the hapten/prohapten hypothesis (Uetrecht, 2007); (2) the pharmacological interaction with immune receptors, the $p-i$ hypothesis (Pichler et al., 2006); (3) the danger model, which is complementary of the hapten hypothesis, adding a secondary signal (e.g. an infection) (Pirmohamed et al., 2002), and (4) the altered peptide model (Illing et al., 2012; Mallal and Phillips, 2012; Norcross et al., 2012; Ostrov et al., 2012). The hapten hypothesis considers that drugs or their metabolites are too small to be immunogenic. Instead, they can bind irreversibly to proteins, generating covalent adducts, which act as antigens (Uetrecht, 2007). The $p-i$ hypothesis advocates a non-covalent interaction between the drug and the HLA protein, implying the formation of neo-antigen determinants, without the involvement of the proteasome. The most recent evidence that HLA-B*5701 is able to lodge abacavir led to the proposal of the altered peptide model, suggesting that binding of the parent drug per se can influence the repertoire of self-peptide ligands to be presented and trigger abacavir-induced HSR (Chessman et al., 2008; Yang et al., 2009; Bharadwaj et al., 2012; Illing et al., 2012; Mallal and Phillips, 2012; Ostrov et al., 2012).

Regardless of the specific mechanism of abacavir-induced HSR, it is noteworthy that the immunogenic effects of the drug are dosedependent (Adam et al., 2012; Norcross et al., 2012; Ostrov et al., 2012). This correlation suggests that abacavir metabolism could be involved. Therefore, a clarification of the drug's metabolic pathways is essential to fully comprehend the mechanisms of abacavir toxicity.

The molecular mechanisms of abacavir-induced cardiotoxicity are even less understood. Although an immune-mediated response can be envisioned, metabolic activation of abacavir to aldehyde intermediates might play a significant role in this context. This hypothesis is consistent with the fact that several aldehydes have been associated with the inception of cardiovascular pathologies. For instance, acetaldehyde, the primary ethanol metabolite, is thought to be involved in the onset of alcoholic cardiomyopathy (Guo and Ren, 2010a). The report of an exacerbated ethanol-induced myocardial contractile dysfunction following acute ethanol exposure, in a murine model with cardiac-specific overexpression of alcohol dehydrogenase (ADH) (Guo and Ren, 2010b), suggests that heart metabolism to aldehydes, and subsequent site-specific protein modification, may underlie xenobiotic-induced cardiotoxicity. Similarly, exposure to the highly reactive $\alpha, \beta$-unsaturated aldehyde acrolein, an endogenous product of lipid peroxidation and ubiquitous environmental pollutant, has been shown to cause myocardial dysfunction (Luo et al., 2007).

Abacavir is a guanosine analog, which is converted intracellularly to the pharmacologically active metabolite, carbovir triphosphate (CBV-TP), via stepwise anabolism. Abacavir is initially phosphorylated to abacavir monophosphate (ABC-MP) by adenosine phosphotransferase, followed by deamination via a cytosolic enzyme to form carbovir monophosphate (CBV-MP). Then two subsequent phosphorylations occur, to the diphosphate by guanylate kinase, and finally to carbovir triphosphate (CBV-TP) by nucleoside diphosphate kinase (Scheme 1). The active metabolite competes with endogenous 2 -deoxyguanosine triphosphate for the nucleic acid chain and, upon incorporation, terminates the DNA chain extension (Faletto et al., 1997; Hervey and Perry, 2000). Concurrently with this activation process abacavir is extensively metabolized by the liver. Primary abacavir metabolism pathways comprise Phase II glucuronidation, mediated by uridine diphosphate glucuronyltransferase and yielding an inactive glucuronide metabolite (ABC-glucuronide), and Phase I oxidation, mediated by $\mathrm{ADH}$ and yielding a carboxylate (ABC-carboxylate). These metabolites are excreted primarily in the urine, where in combination they account for $66 \%$ of the dose; an additional $15 \%$ of the dose is converted into a number of minor metabolites (McDowell et al., 1999), but structural considerations suggest that none of those identified to date are potentially reactive intermediates. By contrast, abacavir metabolism to the carboxylate involves a two-step oxidation process, via a reactive aldehyde intermediate (Walsh et al., 2002). The formation of isomers of both the acid metabolite and the parent drug through ADH metabolism led Walsh et al. (2002) to propose a metabolic pathway involving double bond migration and epimerization processes upon formation of two putative aldehyde intermediates: an unconjugated aldehyde and a conjugated aldehyde (Scheme 1 ).

We have demonstrated previously that the conjugated aldehyde is capable of reacting with proteins in vitro (Charneira et al., 2011) and in rats (Charneira et al., 2012) but, so far, this metabolite has eluded detection in humans. The present work was aimed at identifying abacavir-hemoglobin adducts in HIV-infected patients as biomarkers of abacavir bioactivation to an aldehyde metabolite.

\section{Methods}

\subsection{Chemicals and standards}

All reagents were purchased from Sigma-Aldrich Química S.A. (Madrid, Spain) and used as received. The abacavir-valine Edman adduct standard was synthesized and characterized as described in Charneira et al. (2011).

\subsection{Study design}

The protocol received prior approval from the Ethics Committee of Centro Hospitalar de Lisboa Central, EPE and patients gave their written informed consent. All eligible patients were adults with documented HIV-infection who had received continuous treatment with abacavir-based combined antiretroviral therapy ( $300 \mathrm{mg}$ twice daily or $600 \mathrm{mg}$ once daily) for more than 7 days, regardless of past antiretroviral history. Exclusion criteria were being under 18 years of age, having AIDS-defining conditions and compliance issues. The patients' age, sex, ethnicity, abacavir schedule and antiretroviral co-medication data were recorded. Two healthy volunteers were recruited as controls.

\subsection{Isolation of N-terminal valine-abacavir adducts from hemoglobin}

The blood samples $(2 \mathrm{~mL}$ ) were centrifuged at $3000 \times \mathrm{g}$ for $10 \mathrm{~min}$, to separate plasma from the red blood cells. Cells $(400 \mu \mathrm{L})$ were washed three times with 1 volume of $0.9 \% \mathrm{NaCl}$ after being heated for $60 \mathrm{~min}$ at $60{ }^{\circ} \mathrm{C}$ for HIV inactivation. Hemoglobin precipitation and $\mathrm{N}$-alkyl Edman degradation to detach $\mathrm{N}$-termina valine-abacavir adducts from the protein were performed as described in Charneira et al. (2011).

\subsection{Liquid chromatography-electrospray ionization-tandem mass spectrometry (LC-ESI-MS/MS)}

LC-ESI-MS/MS analyses were performed with a 500-MS LC Ion Trap mass spectrometer (Varian, Inc., Palo Alto, CA). The samples were separated on a Luna C18 (2) column $(250 \mathrm{~mm} \times 4.6 \mathrm{~mm} ; 5 \mu \mathrm{m}$; Phenomenex, Torrance, CA). The injection volume was $20 \mu \mathrm{L}$. The mobile phase was delivered at a flow rate of $200 \mu \mathrm{L} / \mathrm{min}$, using a 17 -min linear gradient from 0 to $100 \%$ acetonitrile, followed by a 3 -min isocratic elution with acetonitrile and a 3 -min linear gradient back to $100 \%$ of $0.1 \%$ 


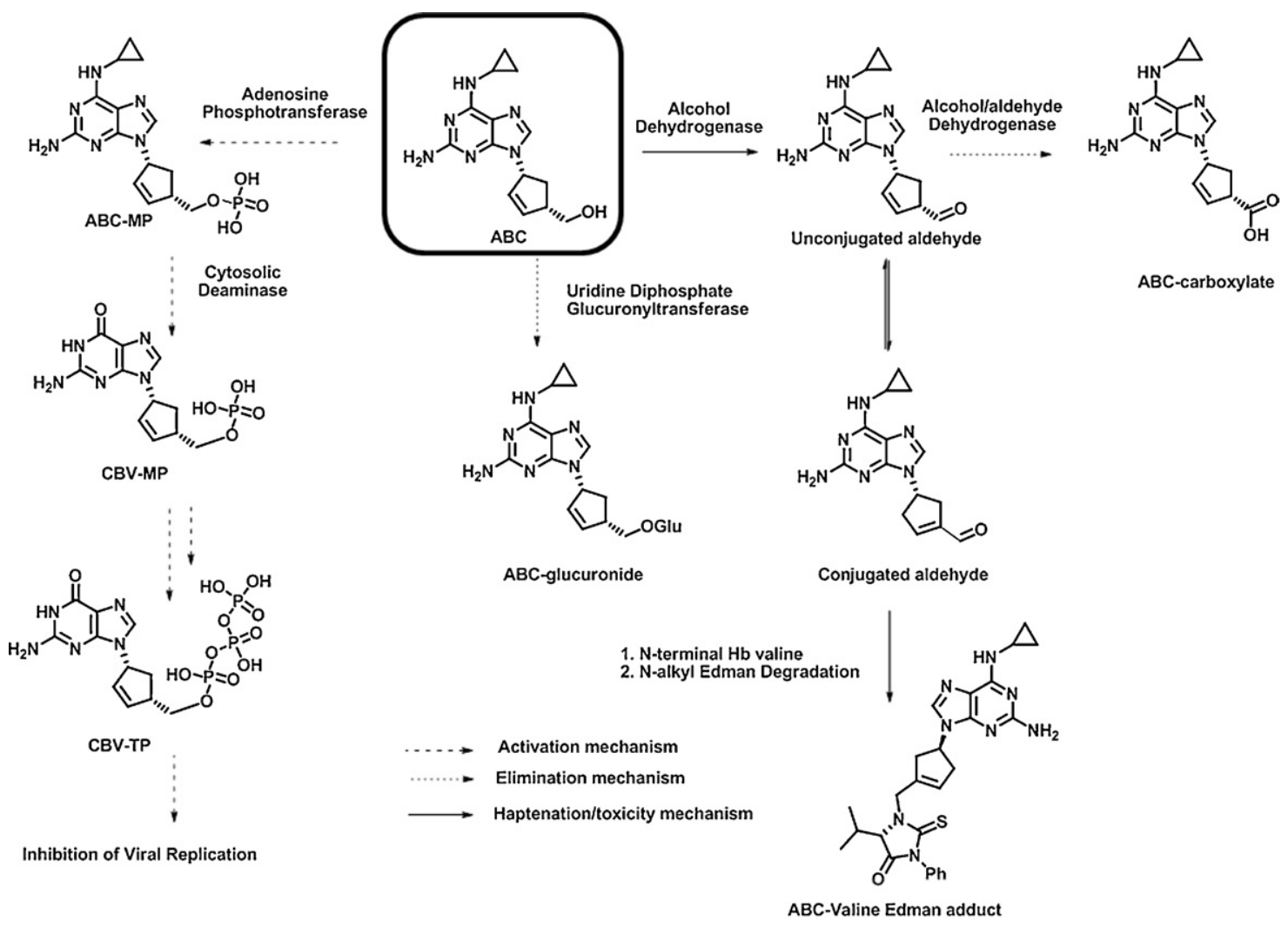

Scheme 1. Metabolic pathways of the anti-HIV drug abacavir.

aqueous formic acid. The system was re-equilibrated for $10 \mathrm{~min}$ before the next injection. Positive ion ESI tandem mass spectrometric analyses were conducted using collision-induced dissociations (CID, up to $\mathrm{MS}^{3}$ ). The experimental parameters were adjusted for maximum sensitivity and structural information on the adducts of interest.

\section{Results}

To obtain evidence for abacavir bioactivation to a reactive aldehyde metabolite in humans, the presence of abacavir adducts with the $\mathrm{N}$-terminal valine of hemoglobin was investigated in ten HIVinfected patients ( 5 men and 5 women; $34-83$ years old; $560 \pm 280$ $\mathrm{CD}_{4}{ }^{+}$cells $\mathrm{mm}^{-3}$; Table 1$)$.

The $N$-alkyl Edman procedure was used for specific detachment of adducts from hemoglobin. LC-ESI-MS/MS analysis of the $\mathrm{MS}^{3}$ transition of the protonated molecule $[\mathrm{m} / \mathrm{z} 503 \rightarrow 313 \rightarrow 235]$ (Fig. 1A) allowed the unequivocal identification of the $N$-terminal valine-abacavir adducts in three patients (two men and one woman on $600 \mathrm{mg}$ abacavir once daily). This identification was based upon undistinguishable mass spectra and identical retention times, when compared with the previously prepared synthetic standard (Charneira et al., 2011). Specifically, under the chromatographic conditions used, all three positive samples and the synthetic standard displayed a signal at $10.5 \mathrm{~min}$, whose $\mathrm{MS}^{3} \mathrm{spec}-$ trum consistently presented a characteristic fragment ion at $\mathrm{m} / \mathrm{z}$ 235 (Fig. 1B). This signal was absent from the control human samples (Fig. 1C) but was identical to that of the synthetic standard (Fig. 1D). Based upon calibration with the standard, the limit of quantification was estimated at $35.6 \mathrm{fmol}$ of adduct on column. The adduct levels were $2.5-7.0 \mathrm{fmol} / \mathrm{mg}$ hemoglobin in the positive samples (Table 1). As persistent inflammation and immune suppression play a crucial role in both HSR and myocardial infarction (MI) (Linneberg et al., 2012; Worm et al., 2010; Ozben and Erdogan, 2008) we considered important to assess the patients'
CD4 count. However, no correlation was found between adduct detection and CD4 count. The time of exposure to abacavir was also considered (Table 1), to search for potential correlations between this factor and increased abacavir adduct levels. However, no significant differences were found between patients with and without detectable adducts $(4.3 \pm 2.5$ years versus $3.3 \pm 2.2$ years of abacavir exposure, respectively). It is conceivable that a larger cohort would be required to clarify this issue.

\section{Discussion}

The present work was aimed at identifying abacavir-hemoglobin adducts in HIV-infected patients as biomarkers of abacavir bioactivation to an aldehyde metabolite. Aldehydes are often implicated in hypersensitivity conditions due to their ability to modify proteins (O'Brien et al., 2005); this, and the fact that $\mathrm{ADH}$ is present in epithelial tissues, including the skin (Lockley et al., 2005), strongly supports the involvement of aldehyde metabolites in abacavir-induced skin toxicity. Likewise, the association between abacavir exposure and increased risk of MI (Costagliola et al., 2010; Islam et al., 2012) is consistent with aldehyde-mediated cardiotoxicity (Guo and Ren, 2010b). Thus, the unquestionable toxicological significance of aldehyde intermediates (O'Brien et al., 2005) along with the fact that several aldehydes (e.g. acetaldehyde and acrolein) have been associated with the inception of cardiovascular pathologies (Guo and Ren, 2010a,b; Luo et al., 2007; Strubelt et al., 1990) justifies the need to investigate causal relationships between abacavir biotransformation to a reactive aldehyde metabolite and the occurrence of abacavir-induced MI in human patients. Hence, whereas the aldehyde levels formed after chronic exposure to abacavir will be much lower than those formed after alcohol abuse, HIV-infected patients have multiple factors that can contribute to ignite the abacavir-associated MI risk. Examples of those factors are: (1) 
Table 1

Patients' demographic and clinical data and abacavir-derived Edman adduct levels.

\begin{tabular}{|c|c|c|c|c|c|c|c|c|}
\hline Patient & Age (years) & $\operatorname{Sex}^{\mathrm{a}}$ & Ethnicity $^{\mathrm{b}}$ & $\begin{array}{l}\mathrm{CD}^{+} \text {count } \\
\left(\text { cell } \mathrm{mm}^{-3}\right)\end{array}$ & $\begin{array}{l}\text { Abacavir } \\
\text { schedule }^{c}\end{array}$ & $\begin{array}{l}\text { Abacavir exposure } \\
\text { (years) }\end{array}$ & cART $^{d}$ & $\begin{array}{l}\text { Abacavir-valine Edman } \\
\text { adducts (fmol/mg } \\
\text { hemoglobin) }^{\mathrm{e}}\end{array}$ \\
\hline A & 41 & M & $\mathrm{C}$ & 1019 & $600 \mathrm{OD}$ & 7 & $3 \mathrm{TC}+\mathrm{PI} / \mathrm{r}$ & 7.0 \\
\hline B & 42 & $\mathrm{~F}$ & $\mathrm{C}$ & 664 & $600 \mathrm{OD}$ & 2 & $3 \mathrm{TC}+\mathrm{PI} / \mathrm{r}$ & 2.5 \\
\hline C & 56 & M & $\mathrm{C}$ & 292 & 600 OD & 4 & $3 \mathrm{TC}+\mathrm{TDF}+\mathrm{PI} / \mathrm{r}$ & 3.3 \\
\hline $\mathrm{D}$ & 38 & M & $\mathrm{C}$ & 711 & 300 BID & 1 & $3 \mathrm{TC}+\mathrm{EFV}$ & $N D^{f}$ \\
\hline $\mathrm{E}$ & 58 & $\mathrm{~F}$ & $\mathrm{C}$ & 236 & 300 BID & 1 & $3 \mathrm{TC}+\mathrm{PI} / \mathrm{r}$ & ND \\
\hline $\mathrm{F}$ & 34 & $\mathrm{~F}$ & A & 684 & 600 OD & 5 & $3 \mathrm{TC}+\mathrm{TDF}+\mathrm{PI} / \mathrm{r}$ & ND \\
\hline G & 70 & $\mathrm{~F}$ & $\mathrm{C}$ & 152 & $600 \mathrm{OD}$ & 5 & $3 \mathrm{TC}+\mathrm{TDF}+\mathrm{PI} / \mathrm{r}$ & ND \\
\hline $\mathrm{H}$ & 38 & $\mathrm{~F}$ & $\mathrm{C}$ & 376 & 600 OD & 1 & $3 \mathrm{TC}+\mathrm{TDF}+\mathrm{EFV}$ & ND \\
\hline I & 37 & M & $\mathrm{C}$ & 682 & $600 \mathrm{OD}$ & 4 & $3 \mathrm{TC}+\mathrm{EFV}$ & ND \\
\hline $\mathrm{J}$ & 83 & M & C & 748 & $600 \mathrm{OD}$ & 6 & $3 \mathrm{TC}+\mathrm{TDF}+\mathrm{PI} / \mathrm{r}$ & ND \\
\hline
\end{tabular}

a $\mathrm{F}$, female; $\mathrm{M}$, male

b C, caucasian; A, african.

c OD - once a day; BID - twice a day.

d cART, combined antiretroviral therapy; 3TC, lamivudine; EFV, efavirenz; and PI/r, boosted protease inhibitor.

e Hemoglobin was precipitated and subjected to $\mathrm{N}$-alkyl-Edman degradation (Charneira et al., 2011). The abacavir-valine Edman adducts were identified and quantified by LC-ESI-MS/MS as described in Section 2.

${ }^{\mathrm{f}} \mathrm{ND}$, not detected.

the persistent inflammatory/infectious and immune suppressive status (Deeks and Phillips, 2009; Linneberg et al., 2012; Ozben and Erdogan, 2008); (2) the glutathione depletion status (De Quay et al., 1992); (3) pharmacogenetic factors (Peters et al., 2005); (4) alcohol consumption and drug abuse; and (5) drug-drug interactions.

Aldehydes are short-lived and extremely difficult to detect in vivo, but can form stable covalent adducts with proteins. Adducts with the $N$-terminal valine of hemoglobin are particularly suitable biomarkers of aldehyde formation because (i) the protein is easily accessed; (ii) $\mathrm{N}$-terminal valines are primary sites of reaction with numerous electrophiles; and (iii) a simple and sensitive method for selective detachment of these adducts from the protein (the $\mathrm{N}$-alkyl Edman procedure) is available (Törnqvist et al., 2002).
In the present study we used this strategy to find evidence for the presence of abacavir adducts in the hemoglobin of HIV-positive patients on abacavir-containing regimens. Through comparison with a synthetic standard, we detected the abacavir-valine Edman adduct unambiguously by LC-ESI-MS/MS in three out of ten patients (Fig. 1). Despite this variability between patients, individual characteristics (age, time of exposure to abacavir or CD4 count) or therapies did not influence adduct detection, most likely due to the small sample size. Thus, whereas clarification of the role of bioactivation in abacavir-induced toxicity still requires much effort in the analysis of clinical samples, we believe to have clearly demonstrated that the search for causal relationships between abacavir biotransformation to a reactive aldehyde metabolite and the

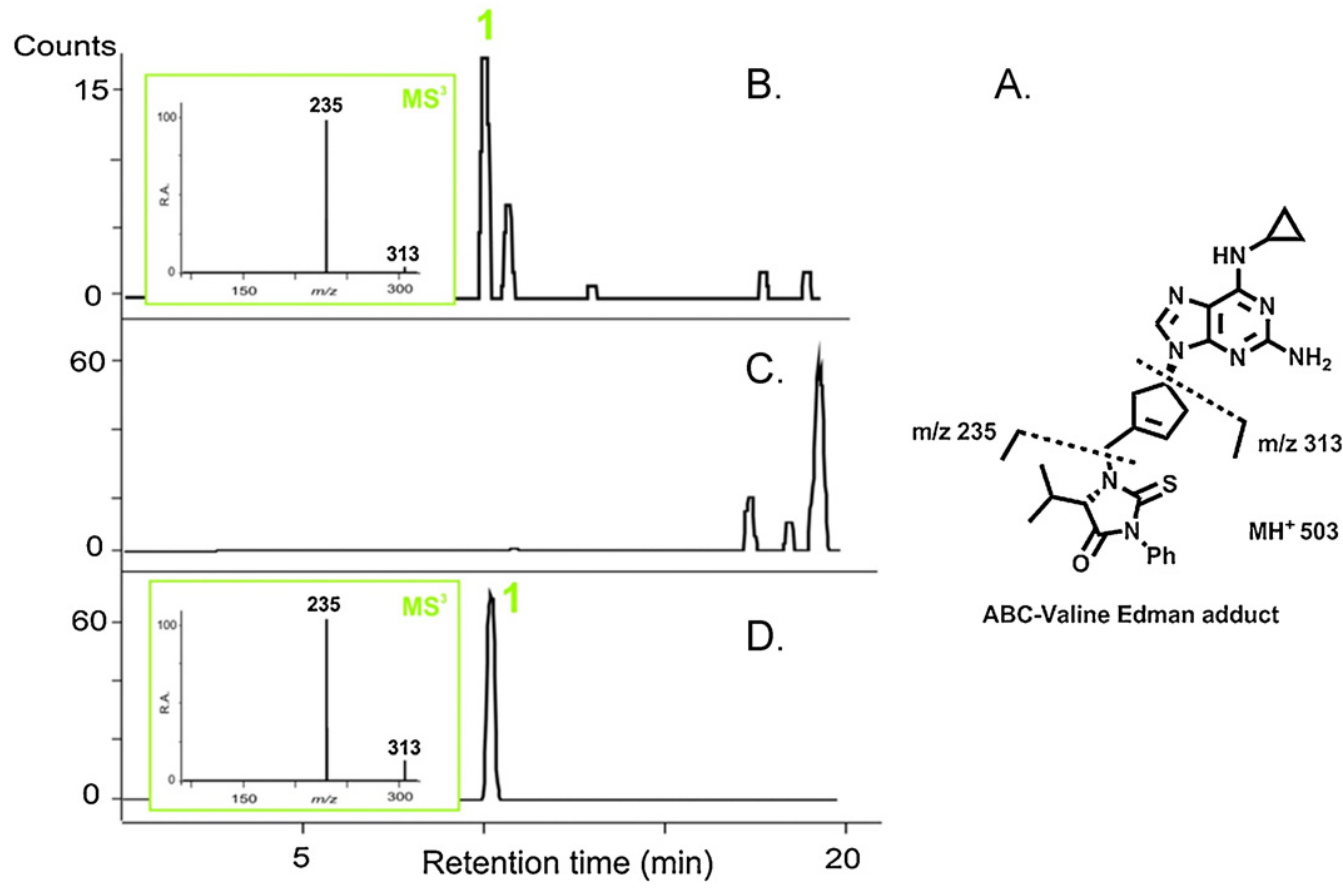

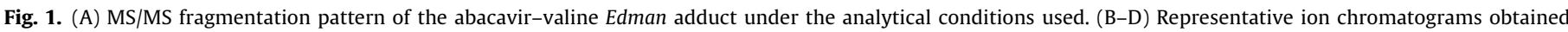

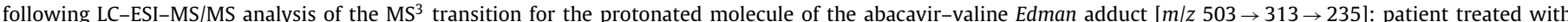

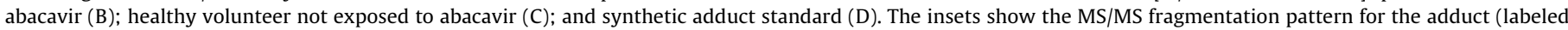

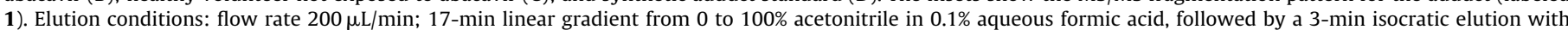
acetonitrile. 
occurrence of abacavir-induced cardiotoxicity in human patients is worth pursuing. Hence these results represent the first report on the involvement of a conjugated aldehyde in the metabolic activation of abacavir in humans.

Furthermore, the fact that only $30 \%$ of the patients had detectable adduct levels implies a high degree of inter-individual variability in abacavir activation/detoxification, which is consistent with the reports of differential susceptibilities to the adverse effects of the drug (Nolan, 2009) among carriers of the high-risk HLA-B*57:01 allele for HSR. Further work is required to clarify the implications of this interpatient variability for cardiotoxicity. Toward this end, we intend to conduct subsequent studies with larger cohorts, to correlate adduct formation/concentration with markers of heart function (routine cardiac imaging studies and blood biomarkers). In addition, an animal model approach, aimed at the evaluation of other cardiac parameters, will also be considered, with the ultimate goal of clarifying the role of covalent adducts in abacavir-induced cardiotoxicity and assess these adducts as biomarkers of cardiotoxicity.

\section{Conclusion}

Taken together, the results reported herein demonstrate that the search for causal relationships between the formation of abacavirderived protein adducts and the occurrence of abacavir-induced toxic events in human patients is worth pursuing. Moreover, the adduct characterization methodology described in the present study is simple, sensitive, accurate, reliable, and applicable to high throughput analyses, as required for testing clinical samples. As such, it is of significant worth for further toxicological studies.

\section{Role of the funding source}

This work was supported by Fundação para a Ciência e a Tecnologia (FCT, Portugal), through research grants (PTDC/SAUTOX/111663/2009 and PTDC/QUI-QUI/113910/2009), and pluriannual funds to Centro de Química Estrutural (PEstOE/QUI/UI0100/2011).

\section{Conflict of interest statements}

The authors declare that there are no conflicts of interest.

\section{Acknowledgement}

Thanks are due to the Portuguese MS Network (IST-UTL Center).

\section{References}

Adam, J., Eriksson, K.K., Schnyder, B., Fontana, S., Pichler, W.J., Yerly, D., 2012. Avidity determines T-cell reactivity in abacavir hypersensitivity. European Journal of Immunology 42, 1706-1716.

Bharadwaj, M., Illing, P., Theodossis, A., Purcell, A.W., Rossjohn, J., McCluskey, J., 2012. Drug hypersensitivity and human leukocyte antigens of the major histocompatibility complex. Annual Review of Pharmacology and Toxicology 52, 401-431.

Charneira, C., Godinho, A.L.A., Oliveira, M.C., Pereira, S.A., Monteiro, E.C., Marques, M.M., Antunes, A.M.M., 2011. Reactive aldehyde metabolites from the anti-HIV drug abacavir: amino acid adducts as possible factors in abacavir toxicity. Chemical Research in Toxicology 24, 2129-2141.

Charneira, C., Grilo, N.M., Pereira, S.A., Godinho, A.L.A., Monteiro, E.C., Marques, M.M., Antunes, A.M.M., 2012. N-terminal valine adduct from the anti-HIV drug abacavir in rat haemoglobin as evidence for abacavir metabolism to a reactive aldehyde in vivo. British Journal of Pharmacology 167, 1353-1361.

Chessman, D., Kostenko, L., Lethborg, T., Purcell, A.W., Williamson, N.A., Chen, Z., Kjer-Nielsen, L., Mifsud, N.A., Tait, B.D., Holdsworth, R., Almeida, C.A., Nolan, D., Macdonald, W.A., Archbold, J.K., Kellerher, A.D., Marriott, D., Mallal, S., Bharadwaj, M., Rossjohn, J., McCluskey, J., 2008. Human leukocyte antigen class I-restricted activation of CD8+ T cells provides the immunogenetic basis of a systemic drug hypersensitivity. Immunity $28,822-832$.
Costagliola, D., Lang, S., Mary-Krause, M., Boccara, F., 2010. Abacavir and cardiovascular risk: reviewing the evidence. Current HIV/AIDS Reports 7, 127-133.

Deeks, S.G., Phillips, A.N., 2009. HIV infection, antiretroviral treatment, ageing, and non-AIDS related morbidity. British Medical Journal 338, a3172.

De Quay, B., Malinverni, R., Lauterburg, B.H., 1992. Glutathione depletion in HIVinfected patients: role of cysteine deficiency and effect of oral N-acetylcysteine. AIDS 6, 815-819.

Faletto, M.B., Miller, W.H., Garvey, E.P., St Clair, M.H., Daluge, S.M., Good, S.S. 1997. Unique intracellular activation of the potent anti-human immunodeficiency virus agent 1592U89. Antimicrobial Agents and Chemotherapy 41, 1099-1107.

FDA alert, 2011. Abacavir - Ongoing Safety Review: Possible Increased Risk of Heart Attack. http://www.drugs.com/fda/abacavir-ongoing-safety-reviewpossible-increased-risk-heart-attack-12914.html (accessed 22.01.13).

Guo, R., Ren, J., 2010a. Alcohol and acetaldehyde in public health: from marvel to menace. International Journal of Environmental Research and Public Health 7, 1285-1301.

Guo, R., Ren, J., 2010b. Alcohol dehydrogenase accentuates ethanol-induced myocardial dysfunction and mitochondrial damage in mice: role of mitochondrial death pathway. PLoS One 5, e8757.

Hervey, P.S., Perry, C.M., 2000. Abacavir: a review of its clinical potential in patients with HIV infection. Drugs 60, 447-479.

Illing, P.T., Vivian, J.P., Dudek, N.L., Kostenko, L., Chen, Z., Bharadwaj, M., Miles, J.J., Kjer-Nielsen, L., Gras, S., Williamson, N.A., Burrows, S.R., Purcell, A.W., Rossjohn, J., McCluskey, J., 2012. Immune self-reactivity triggered by drug-modified HLApeptide repertoire. Nature 486, 554-558.

Islam, F., Wu, J., Jansson, J., Wilson, D.P., 2012. Relative risk of cardiovascular disease among people living with HIV: a systematic review and meta-analysis. HIV Medicine 13, 453-468.

Linneberg, A., Jacobsen, R.K., Jespersen, L., Abildstrøm, S.Z., 2012. Association of subcutaneous allergen-specific immunotherapy with incidence of autoimmune disease, ischemic heart disease, and mortality. Journal of Allergy and Clinical Immunology 129, 413-419.

Lockley, D.J., Howes, D., Williams, F.M., 2005. Cutaneous metabolism of glycol ethers. Archives of Toxicology 79, 160-168.

Luo, J., Hill, B.G., Gu, Y., Cai, J., Srivastava, S., Bhatnagar, A., Prabhu, S.D. 2007. Mechanisms of acrolein-induced myocardial dysfunction: implications for environmental and endogenous aldehyde exposure. American Journal of Physiology-Heart and Circulatory Physiology 293, H3673-H3684.

Mallal, S., Phillips, E., 2012. Abacavir and the altered peptide repertoire model: clinical implications. Journal of the International AIDS Society 15 (Suppl. 4), 18119.

McDowell, J.A., Chittick, G.E., Ravitch, J.R., Polk, R.E., Kerkering, T.M., Stein, D.S., 1999. Pharmacokinetics of $\left[{ }^{14} \mathrm{C}\right]$ abacavir, a human immunodeficiency virus type 1 (HIV-1) reverse transcriptase inhibitor, administered in a single oral dose to HIV-1-infected adults: a mass balance study. Antimicrobial Agents and Chemotherapy 43, 2855-2861.

Nolan, D., 2009. HLA-B*5701 screening prior to abacavir prescription: clinical and laboratory aspects. Critical Reviews in Clinical Laboratory Sciences 46, 153-165.

Norcross, M.A., Luo, S., Lu, L., Boyne, M.T., Gomarteli, M., Rennels, A.D., Woodcock, J., Margulies, D.H., McMurtrey, C., Vernon, S., Hildebrand, W.H., Buchli, R., 2012. Abacavir induces loading of novel self-peptides into HLA-B*57:01: an autoimmune model for HLA-associated drug hypersensitivity. AIDS 26, F21-F29.

O’Brien, P.J., Siraki, A.G., Shangari, N., 2005. Aldehyde sources, metabolism, molecular toxicity mechanisms, and possible effects on human health. Critical Reviews in Toxicology 35, 609-662.

Ostrov, D.A., Grant, B.J., Pompeu, Y.A., Sidney, J., Harndahl, M., Southwood, S., Oseroff, C., Lu, S., Jakoncic, J., Oliveira, C.A.F., Yang, L., Mei, H., Shi, L., Shabanowitz, J., English, A.M., Wriston, A., Lucas, A., Phillips, E., Mallal, S., Grey, H.M., Sette, A., Hunt, D.F., Buus, S., Peters, B., 2012. Drug hypersensitivity caused by alteration of the MHC-presented self-peptide repertoire. Proceedings of the National Academy of Sciences of the United States of America 109, 9959-9964.

Ozben, B., Erdogan, O., 2008. The role of inflammation and allergy in acute coronary syndromes. Inflammation \& Allergy - Drug Targets 7, 136-144.

Peters, E.S., McClean, M.D., Liu, M., Eisen, E.A., Mueller, N., Kelsey, K.T., 2005. The ADH1C polymorphism modifies the risk of squamous cell carcinoma of the head and neck associated with alcohol and tobacco use. Cancer Epidemiology, Biomarkers and Prevention 14, 476-482.

Pichler, W.J., Beeler, A., Keller, M., Lerch, M., Posadas, S., Schmid, D., Spanou, Z., Zawodniak, A., Gerber, B., 2006. Pharmacological interaction of drugs with immune receptors: the $\mathrm{p}-\mathrm{i}$ concept. Allergology International 55, $17-25$.

Pirmohamed, M., Naisbitt, D.J., Gordon, F., Park, B.K., 2002. The danger hypothesis-potential role in idiosyncratic drug reactions. Toxicology 181-182, 55-63.

Strubelt, O., Brasch, H., Pentz, R., Younes, M., 1990. Experimental studies on the acute cardiovascular toxicity of formalin and its antidotal treatment. Journal of Toxicology - Clinical Toxicology 28, 221-233.

Törnqvist, M., Fred, C., Haglund, J., Helleberg, H., Paulsson, B., Rydberg, P., 2002. Protein adducts: quantitative and qualitative aspects of their formation, analysis and applications. Journal of Chromatography B 778, 279-308.

Uetrecht, J., 2007. Idiosyncratic drug reactions: current understanding. Annual Review of Pharmacology and Toxicology 47, 513-539. 
Walsh, J.S., Reese, M.J., Thurmond, L.M., 2002. The metabolic activation of abacavir by human liver cytosol and expressed human alcohol dehydrogenase isozymes. Chemico-Biological Interactions 142, 135-154.

Worm, S.W., Sabin, C., Weber, R., Reiss, P., El-Sadr, W., Dabis, F., De Wit, S., Law, M., Monforte, A.D., Friis-Møller, N., Kirk, O., Fontas, E., Weller, I., Phillips, A., Lundgren, J., 2010. Risk of myocardial infarction in patients with HIV infection exposed to specific individual antiretroviral drugs from the 3 major drug classes: the data collection on adverse events of anti-HIV drugs (D:A:D) study. Journal of Infectious Diseases 201, 318-330.

Yang, L., Chen, J., He, L., 2009. Harvesting candidate genes responsible for serious adverse drug reactions from a chemical-protein interactome. PLoS Computational Biology 5, e1000441. 